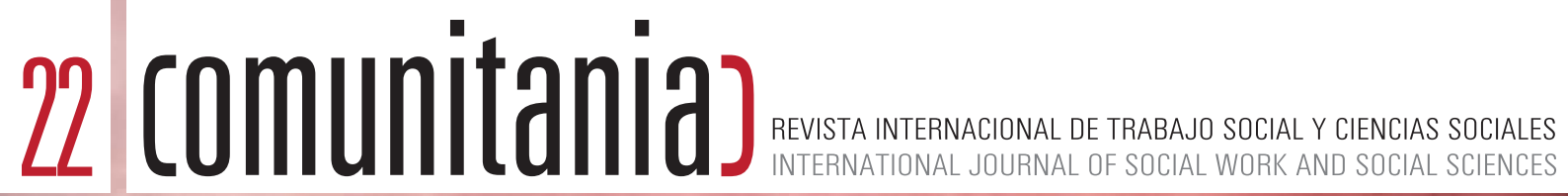

JULIO / 2021

ISSN: 2173-0512 / www.comunitania.com

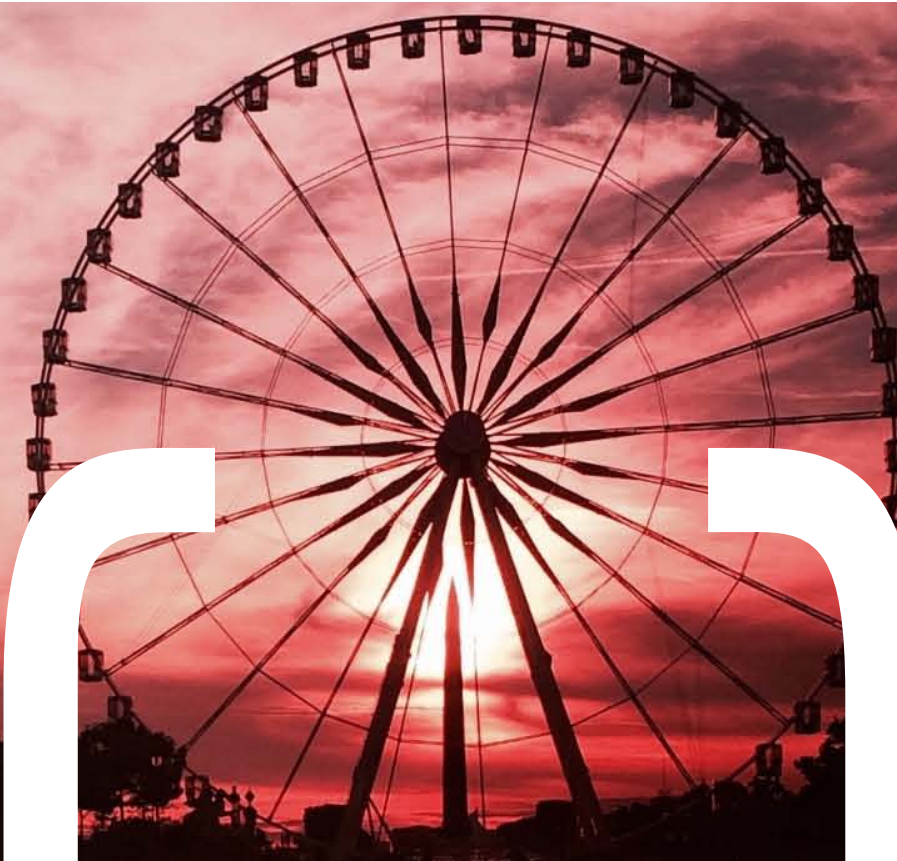




\title{
Programas intergeneracionales que fomentan la participación social de las personas mayores en España. Una mirada desde el Trabajo Social Intergenerational programmes that promote the social participation of older people in Spain. A view from Social Work
}

\author{
Juana María Morcillo Martínez*
}

* Universidad de Jaén. jmmorcil@ujaen.es

\begin{abstract}
:
The present desk research study aims to systematically review, from the discipline of social work, programmes that enhance interaction and exchange between different generations as new opportunities for older people. Intergenerational programmes are among the most effective measures to bring people from different generations together, promote quality of life and are established as a means of empowerment in communities. The methodological process was carried out through a literature search in the Social Services Abstracts database providing bibliographic coverage of current research on the development of intergenerational programmes related to social work and social services. The results point to a lack of research on the study of the impact of intergenerational programmes that contribute to fostering the social participation of older people, between 2015 and 2020, in Spain.
\end{abstract}

Keywords: personas mayores; participación social; programas intergeneracionales; envejecimiento activo; trabajo social.

\section{Resumen:}

El presente estudio de investigación documental tiene como objetivo revisar sistemáticamente, desde la disciplina del trabajo social, los programas que acrecientan la interacción y el intercambio entre diferentes generaciones como nuevas oportunidades para las personas mayores. Los programas intergeneracionales se sitúan entre las medidas más eficaces para unir a personas que pertenecen a diferentes generaciones, promueven la calidad de vida y se establecen como vía de empoderamiento en las comunidades. El proceso metodológico se llevó a cabo a través de una búsqueda bibliográfica en la base de datos Social Services Abstracts proporcionando cobertura bibliográfica de investigaciones actuales sobre el desarrollo de programas intergeneracionales relacionados con el trabajo social y los servicios sociales. Los resultados señalan la carencia de investigaciones que se preocupen por el 
estudio del impacto de los programas intergeneracionales que contribuyan a fomentar la participación social de las personas mayores, entre los años 2015 y 2020, en España.

Palabras clave: older people; social participation; intergenerational programmes; active ageing; social work.

\section{Article info:}

Received: 21/04/2021 / Received in revised form: 21/04/2021

Accepted: 08/06/2021 / Published online: 31/06/2021

DOI: http://dx.doi.org/10.5944/comunitania.22.5

\section{Tntroducción}

El envejecimiento de las personas junto con la dependencia, como incapacidad funcional, no es un fenómeno nuevo en España. La atención a las personas mayores, la promoción de su autonomía personal y su participación social queda constituida como uno de los principales retos en la intervención desde la disciplina del trabajo social. Se define la participación social como iniciativa social en las que las personas toman parte consciente en un espacio, posicionándose y sumándose a otros grupos sociales (Freie Universität Berlín, 2021). La participación social se entiende en la actualidad como la inclusión de distintos actores sociales en determinados ámbitos sociales (IMSERSO, 2018). En este sentido, la participación social de las personas mayores es un proceso necesario que contribuye a mejorar su calidad de vida y donde es fundamental visibilizar su aportación de forma eficaz y eficiente en la sociedad.

Por otro lado, y como datos a tener en cuenta, en España, entre 1999 y 2020, la esperanza de vida de la población masculina ha pasado de 75,4 a 80,9 años y la de la población femenina de 82,3 a 86,2 años, según los indicadores demográficos básicos que publica el INE (2020). Las mujeres ocupan el primer puesto, casi dos tercios de la población mayor de 65 años son mujeres. La diferencia en años de esperanza de vida por parte del colectivo femenino frente al colectivo masculino creció hasta mediados de los años noventa, como consecuencia de una mortalidad masculina más alta debido a estilos de vida menos saludables, señalando que la diferencia a favor de la mujer se ha ido reduciendo en las últimas dos décadas (INE,2020). En la actualidad, la pirámide de población de España continúa su proceso de envejecimiento (INE, 2020; IGN, 2020; CSIC, 2019). Junto a ello, el aumento de la esperanza de vida constituye uno de los indicadores que mejor representa la buena salud de la población mayor española.

Ha de tenerse en cuenta que uno de los retos más importantes que afrontan, en la actualidad, las Administraciones en España es el diseño y ejecución de políticas sociales de envejecimiento activo adaptado a las necesidades reales que tiene este colectivo. (Gobierno de España, 2019; IMSERSO, 2017; OMS, 2018). El aumento de la población de personas mayores, unido a cambios sustanciales en las formas de 
organización y reorganización de la familia y del papel fundamental que desarrollan las personas cuidadoras (la mayoría mujeres) han situado a la dependencia y a la vejez en el punto de mira de las políticas sociales en el Estado español.

El envejecimiento es la característica principal de la población de los países desarrollados, que se inició hace varias décadas en Europa. En general, esta tendencia se visibiliza en la evolución que sufrió la estructura de edad de la población y se refleja en el aumento de la proporción de personas mayores junto con una caída en el colectivo de personas en edad laboral en el total de la población (Eurostat, 2020). Los países conseguirán afrontar el envejecimiento de su población si sus gobiernos, organizaciones implicadas en esta temática y la sociedad en su conjunto, consensuan acciones relacionadas con el desarrollo de políticas y programas de envejecimiento activo que mejoren la salud y la participación plena y efectiva de las personas mayores en su comunidad. Del mismo modo, en los tiempos actuales, los países en vías de desarrollo deben considerar cambios importantes en la orientación de sus políticas públicas, sobre todo en lo relacionado con la mejora del sistema de pensiones, la vivienda y los sistemas sociosanitarios para la atención geriátrica (OMS, 2015).

La Organización Mundial de la Salud (2015), considera que la salud, la participación y la seguridad son la base esencial para que las personas disfruten de una óptima calidad de vida conforme van envejeciendo. Y, considera el envejecimiento activo como un proceso para lograr el objetivo de optimizar la calidad de vida y la esperanza de vida saludable a medida que la persona se hace mayor. En este sentido, el concepto de la OMS está construido sobre tres pilares fundamentales relacionados con: (i) Salud física, mental y social, (ii) Participación: relacionada con los derechos y empoderamiento en cuestiones sociales, económicas, culturales, etc. (iii) Seguridad: relacionada con protección, dignidad y asistencia.

El envejecimiento y la vejez tiene consecuencias sociales (Carabaña, 2003; Zafra, Peiró, Ramón, Álvarez-Dartel y Borrell, 2006). Desde el punto de vista social el envejecimiento de la población plantea cambios en el ámbito laboral, en las familias y las relaciones intergeneracionales. Para responder con éxito a los retos de una población que envejece han de diseñarse actividades en las que participen todas las generaciones.

Desde la disciplina del trabajo social se interviene a nivel individual, grupal y comunitario con la finalidad de conseguir la participación plena de las personas en la sociedad. Siguiendo a diferentes autores el empoderamiento es una acción integradora, holística y adecuada a las necesidades de la población en una posición vulnerable o en riesgo de exclusión social, pudiendo ser dirigida a diferentes grupos de personas (Lee, 2001; Adams, 2008; Faleiros, 2003 y Folgheraiter, 2011).

Como introducción a la cuestión de las relaciones intergeneracionales es transcendental hablar de las generaciones, las cuales han sido objeto de estudio a lo largo de la historia en diferentes disciplinas como la sociología, la literatura o la filosofía, entre 
otras (Lain, 1945; Donati, 1999; Martin, 2008; Leccardi y Feixa, 2011; Caballero y Baigorri, 2013). Las relaciones intergeneracionales y la condición humana quedan intrínsecamente relacionadas. Sin embargo, la distancia entre generaciones es una realidad que determina a la sociedad actual, donde el envejecimiento de la población, cada vez más en auge, debe obligar al desarrollo de nuevas formas de solidaridad intergeneracional. Así mismo, entendemos que programas intergeneracionales son los relacionados con formas de creación de espacios para el encuentro, la promoción del apoyo social y el intercambio de valores encaminados a producir entre las distintas generaciones cambios positivos y beneficios individuales y comunitarios que permitan la construcción de una sociedad más justa, integradora y solidaria" (IMSERSO, 2010). Los programas intergeneracionales se sitúan entre las medidas más pertinentes para unir generaciones, puesto que la investigación ha demostrado que los mismos ofrecen múltiples oportunidades para promover la participación social y la calidad de vida en el individuo (Gutiérrez y Hernández, 2013; Lambert y Fruit, 1990; MacCallum, Palmer, Wright, Cumming-Potvin, Northcote, Booker y Tero, 2006).

Desde el trabajo social se considera que una manera eficaz y emergente para promover el envejecimiento activo y la participación social de las personas mayores debe ir acompañada del intercambio de experiencias entre generaciones donde el apoyo social sea reciproco. En este sentido, El contacto directo entre generaciones produce innegables beneficios para el individuo (Escarbajal de Haro y Martinez de Miguel, 2012; Hank, 2012).

Se define envejecimiento activo como: "proceso de optimización de las oportunidades de salud, participación y seguridad con el fin de mejorar la calidad de vida a medida que las personas envejecen" (OMS, 2002). Siguiendo a Pinazo, Lorente, Limón. Fernández y Bermejo (2010) queda relacionado con:

1. La participación y el empoderamiento de las personas mayores en el ámbito social, económico, político, cultural, educativo y sanitario.

3. Un nuevo modelo de sociedad en que las personas mayores sean protagonistas de su vida y no sean consideradas como meras receptoras de recursos según sus circunstancias.

4. Un enfoque intergeneracional en la comunidad donde se les considere como parte esencial de su entorno.

El desarrollo integral de la persona a lo largo de todo su ciclo vital.

Teniendo en cuenta lo anterior, es evidente que el diseño y ejecución de los programas intergeneracionales tienen un lugar predominante en el envejecimiento activo y calidad de vida de las personas mayores (Braveman, Egeter y Williams, 2011). La interdependencia y la solidaridad intergeneracional son dos principios importantes en la forma de entender el envejecimiento activo como un proceso que sucede en un contexto social de relaciones grupales y comunitarias. Para responder con 
éxito a los retos de una población que envejece han de diseñarse actividades en las que participen todas las generaciones. (Walker, 2006).

Los programas intergeneracionales son un recurso que proporciona que las personas mayores sean consideradas como miembros activos, productivos y valorados en la sociedad. Entre otras cuestiones, sirven para construir vínculos que proporciona a este colectivo un medio de transmisión de conocimientos y sabiduría a las nuevas generaciones, a la vez que les permite seguir manteniéndose como miembros activos de la comunidad. (Pinazo y Kaplan, 2012). De ahí que, en la presente investigación, se revisa sistemáticamente, desde la disciplina del trabajo social y en los últimos cinco años en España, qué actividades y programas intergeneracionales acrecientan la participación social entre personas de diferentes generaciones. El objetivo que se persigue queda relacionado con mejorar la calidad de vida de las personas mayores a través de su participación social.

\section{Método}

\section{Materiales}

En el presente trabajo se ha llevado a cabo la metodología de revisión sistemática como procedimiento metodológico que emplea estrategias científicas con la finalidad de limitar sesgos en el proceso de selección, valoración y síntesis de los estudios relevantes sobre un tema (Goodman, 1996). El diseño del estudio realizado es de tipo exploratorio y descriptivo. Del mismo modo, se ha seguido de forma minuciosa los estándares PRISMA relacionados con el diseño metodológico: protocolo, proceso de búsqueda, selección y síntesis de resultados (Moher, Liberati,Tetzlaff, Altman, \&The PRISMA group, 2009) de estudios relacionados con la participación de las personas mayores en programas intergeneracionales en España, en los últimos cinco años. En la primera fase de la revisión se trabajó con 169 resúmenes seleccionados con las palabras clave de interés para la consecución de nuestro objetivo. En una segunda fase se seleccionaron 6 artículos mediante el análisis de los citados resúmenes, incluyendo solamente los que estudiaran y analizaran nuestro objeto de estudio desde la disciplina y ámbito de intervención del trabajo social.

\section{Procedimiento}

La búsqueda se llevó a cabo en el mes de noviembre de 2020 en la base de datos Social Services Abstracts. Se utilizaron varias combinaciones de términos con boleanos (AND y OR). Los descriptores utilizados fueron: participación social y tercera edad, programas intergeneracionales, programas de envejecimiento activo, solidaridad intergeneracional e interdepenencia, bienestar y personas mayores. Y, los criterios de inclusión han sido aquellos trabajos publicados en inglés o español entre los años 2015 y 2020, en concreto hasta marzo de 2020, fecha en la que se declaró el Estado de 
Alarma en España por Real Decreto-ley 8/2020, de 17 de marzo, que genera medidas extraordinarias para hacer frente al impacto económico y social del COVID-19.

\section{Proceso de selección de los estudios}

En la realización de esta revisión sistemática sólo se seleccionaron aquellos estudios en los que se evidenció que quedaban intrínsecamente relacionados con la intervención desde el ámbito del trabajo social. Fueron finalmente seleccionadas:

Una reflexión teórica y análisis de un caso de acción comunitaria y voluntariado.

1. Un programa que describe un método de atención socioeducativa a través del arte y de la participación.

2. Un análisis descriptivo y multivariado utilizando SPSS 22.0 donde se identifican y codifican 557 enlaces centrados en 7 dimensiones de envejecimiento activo.

3. Un estudio exploratorio relacionado con la participación en organizaciones de personas de la tercera edad en Australia y en España.

4. Un proyecto que incluye la participación de voluntarios virtuales, una parte de la red que promueve su uso en una manera inclusiva y participativa.

5. Un proyecto de investigación cualitativa que analiza el impacto de los programas intergeneracionales desde la escuela y diferentes instituciones sociales

TABLA 1. Descripción de la búsqueda

\begin{tabular}{|l|c|c|c|}
\hline Base de datos & Palabras clave & $\begin{array}{c}\text { Trabajos } \\
\text { encontrados }\end{array}$ & Trabajos incluidos \\
\hline \multirow{5}{*}{$\begin{array}{l}\text { Social Services } \\
\text { Abstract }\end{array}$} & $\begin{array}{c}\text { Programas } \\
\text { intergeneracionales }\end{array}$ & 18 resultados & 2 \\
\cline { 2 - 4 } & $\begin{array}{c}\text { Programas de } \\
\text { envejecimiento activo }\end{array}$ & 6 resultados & 2 \\
\cline { 2 - 4 } & $\begin{array}{c}\text { Solidaridad } \\
\text { intergeneracional e } \\
\text { interdependencia }\end{array}$ & 24 resultados & 2 \\
\cline { 2 - 4 } & $\begin{array}{c}\text { Bienestar y personas } \\
\text { mayores }\end{array}$ & 95 resultados & 0 \\
\cline { 2 - 4 } & $\begin{array}{c}\text { Participación social y } \\
\text { tercera edad }\end{array}$ & 26 resultados & 0 \\
\hline
\end{tabular}

Fuente: elaboración propia.

\section{Resultados}

Se resumen a continuación los principales hallazgos obtenidos de la selección de los artículos incluidos en la revisión sistemática. Fueron finalmente seleccionadas: 


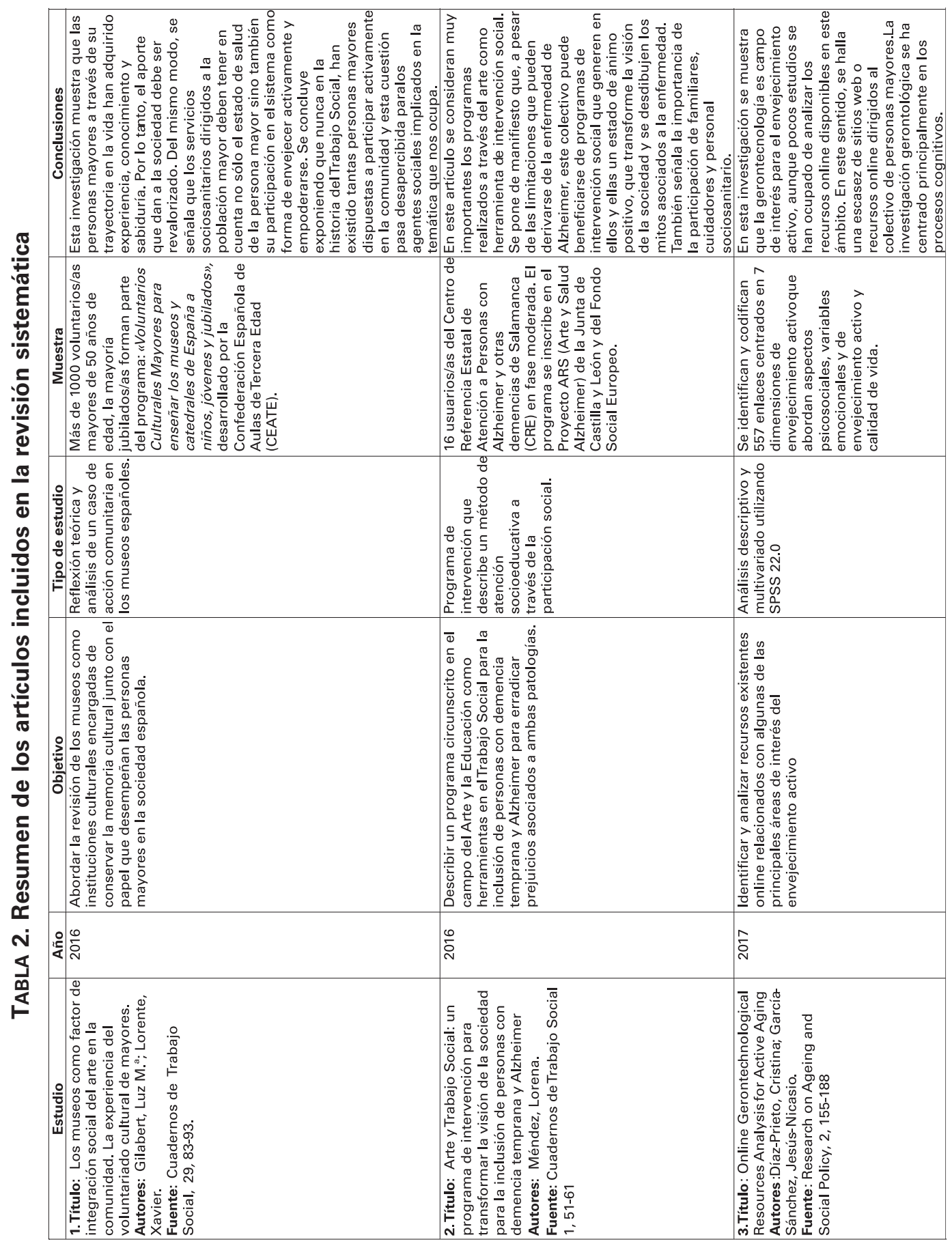




\begin{tabular}{|c|c|c|}
\hline 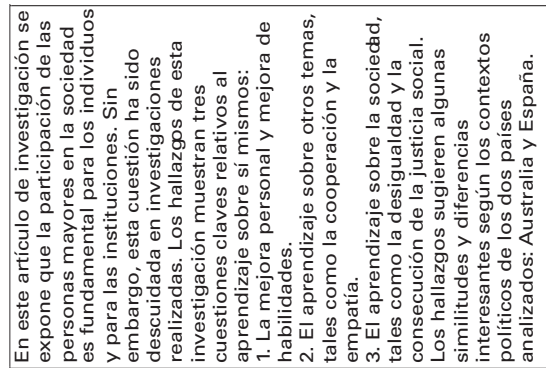 & 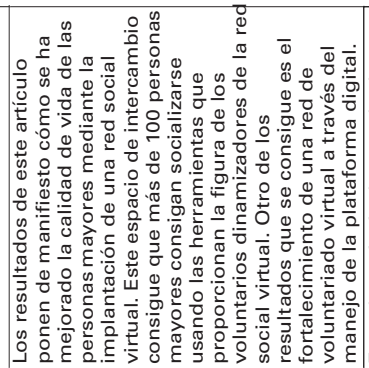 & 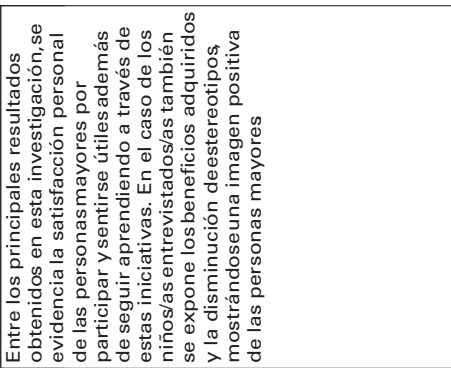 \\
\hline 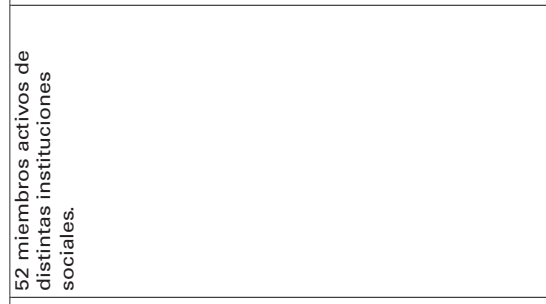 & 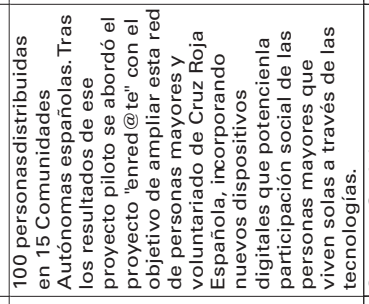 & 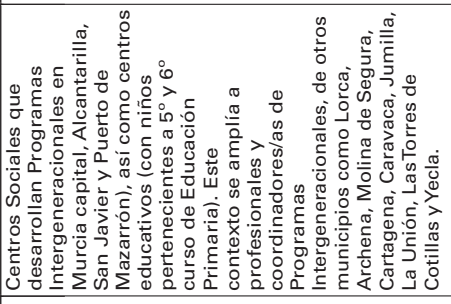 \\
\hline & 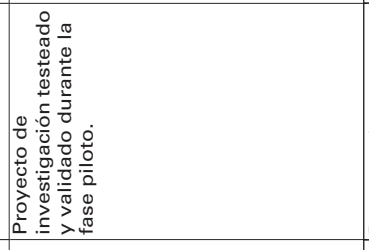 & 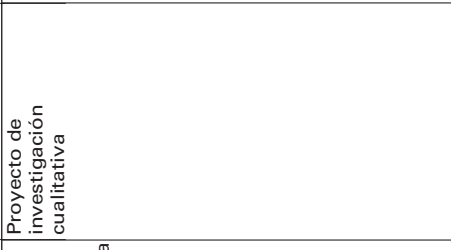 \\
\hline 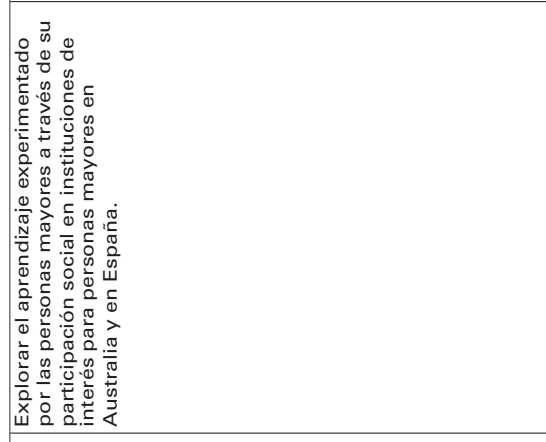 & 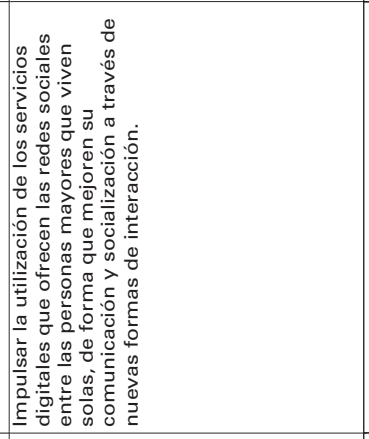 & 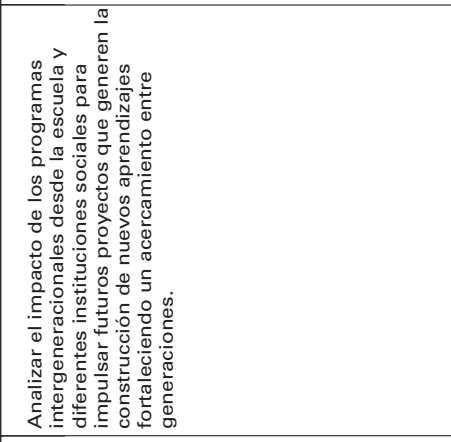 \\
\hline & ¿্র & 冓 \\
\hline 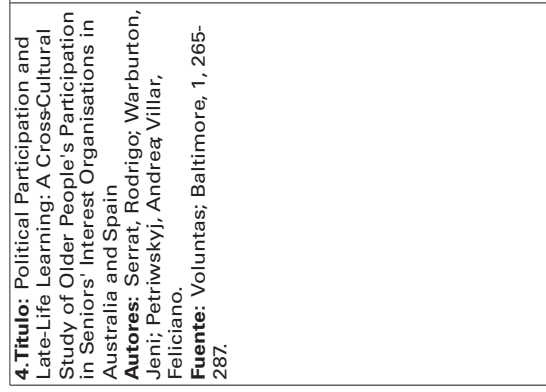 & 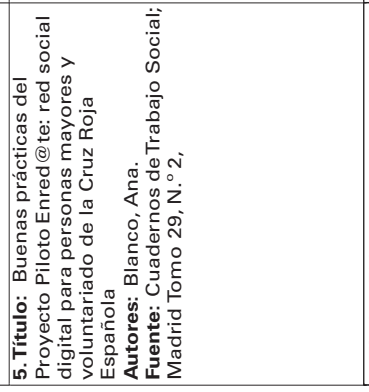 & 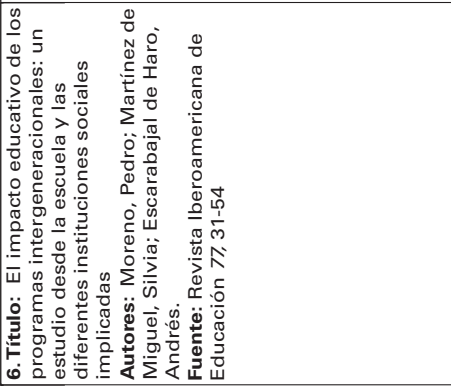 \\
\hline
\end{tabular}




\section{Discusión}

En relación a los hallazgos encontrados señalamos que la investigación muestra cuestiones claves relacionadas con nuestro objeto de estudio: revisar sistemáticamente, desde la disciplina del trabajo social, los programas que acrecientan la interacción y el intercambio entre personas de diferentes generaciones, en España, como nuevas oportunidades para las personas mayores, desde el año 2015 hasta el año. 2020. En este sentido, establecemos las siguientes precisiones:

Primer hallazgo: Reflexión teórica y análisis de un caso de acción comunitaria y voluntariado.

En esta investigación se expone que las personas mayores consideran que realizar voluntariado en un programa intergeneracional les ayuda a mejorar su capacidad funcional y cognitiva (Sánchez y Díaz, 2005). El voluntariado, a través de los programas intergeneracionales, incrementa la cooperación, la interacción y el intercambio de sinergias compartiendo conocimientos y experiencias positivas entre población joven y población mayor. (Ventura-Merkel y Lidoff, 1983). En este sentido, los programas intergeneracionales han de estar diseñados de tal forma que ambas generaciones, sin lazos biológicos, queden implicados en interacciones efectivas donde se proporcionen recursos de apoyo mutuo que provean bienestar, seguridad y calidad de vida a una y otra generación. Así mismo, es necesario que se consigan beneficios sociales, empoderamiento y se apliquen a la comunidad de forma efectiva (Kuehne, 2003; Hatton-Yeo y Ohsako, 2001; McCrea, Weissman y ThorpeBrown, 2004). En este hallazgo se concluye exponiendo que nunca en la historia delTrabajo Social, han existido tantas personas mayores dispuestas a participar activamente en la comunidad y esta cuestión pasa desapercibida para los agentes sociales implicados en la temática que nos ocupa.

Segundo hallazgo: Programa que describe un método de atención socioeducativa a través del arte y de la participación social para erradicar prejuicios en relación a la demencia y al Alzheimer.

En este programa de intervención social se muestra que el impacto socioeducativo en los programas intergeneracionales, en sus diferentes ámbitos de actuación, han de ir encaminados a fomentar la participación social de las personas mayores y erradicar prejuicios vinculados a su edad (Gutiérrez y Hernández, 2013; Lambert y Fruit, 1990; MacCallum, Palmer, Wright, Cumming-Potvin, Northcote, Booker y Tero, 2006). En este hallazgo se consideran muy importantes los programas socioeducativos realizados a través del arte como herramienta de intervención social. Se pone de manifiesto que, a pesar de las limitaciones que pueden derivarse de enfermedades relacionadas con la demencia, las personas mayores han de beneficiarse de programas de intervención social que las empoderen y donde participen familiares y personal sociosanitario. En España, los servicios sociosanitarios dirigidos a la población 
mayor deben tener en cuenta no sólo su estado de su salud, sino también su participación activa con la finalidad de envejecer activamente y empoderarse, teniendo en cuenta que, un gran porcentaje de usuarios y usuarias de los servicios sociosanitarios son las personas mayores (IMSERSO, 2018). Es fundamental la transformación de la visión de la sociedad desdibujándose los mitos asociados al envejecimiento. En este hallazgo se concluye reconociendo la satisfacción personal y empoderamiento de las personas mayores al participar de forma activa y empoderarse, eliminándose estereotipos relacionados con la vejez y el edadismo y reconociéndose una imagen positiva de las personas mayores.

Tercer hallazgo: Un análisis descriptivo y multivariado utilizando SPSS 22.0 donde se identifican y codifican 557 enlaces centrados en 7 dimensiones de envejecimiento activo.

En esta investigación se muestra que la gerontecnología es un campo importante para el envejecimiento activo y pocos estudios se han ocupado de analizar los recursos online disponibles en este ámbito de actuación. La investigación gerontológica se ha centrado principalmente en patologías físicas y cognitivas, hallándose una escasez de sitios web o recursos tecnológicos dirigidos a la población mayor. Por este motivo, el uso de determinados dispositivos en cualquier faceta de la vida de los mayores, así como la facilidad de conectividad y ubicuidad que presentan, está conduciendo a muchos investigadores a analizar procesos de rutinización de la tecnología vinculados al envejecimiento. Por ello, junto con este interés en conocer cómo las personas mayores adoptan y utilizan determinados dispositivos digitales, está también el de afrontar los desafíos que este colectivo presenta en el conocimiento y uso de las TIC (McCreadie yTinker, 2005; Sixsmith, 2013; Woolrych, Gibson, Sixsmith y Sixsmith, 2015). En este hallazgo se pone de manifiesto la necesidad de una mayor implicación institucional, social y científica para aprovechar al máximo las posibilidades que brinda internet, las cuales podrían constituirse como algunas de las causas de la brecha digital de tipo generacional existente en España.

Cuarto hallazgo: Un estudio exploratorio relacionado con la participación en organizaciones de personas de la tercera edad en Australia y en España.

En este estudio se muestra que la participación social de las personas mayores en la sociedad es esencial para las personas y para las instituciones. Sin embargo, esta cuestión ha sido descuidada en numerosas investigaciones realizadas. Del mismo modo, se señala que los profesionales que participan en programas intergeneracionales muestran que la práctica intergeneracional es útil para todos los colectivos implicados en el programa, subrayando cuestiones intrínsecamente relacionadas con la mejora personal y mejora de habilidades sociales. También se pone de manifiesto que este tipo de prácticas contribuyen a acrecentar las redes sociales de las personas mayores, evitando la soledad y el aislamiento. Los trabajos que han hecho alusión a este fenómeno son varios (Ostir, Ottenbacher y Maarkides, 2004; MacCa- 
Ilum et al., 2006). Es fundamental entender que estos programas tienen efectos positivos para la población mayor y otros agentes implicados en la relación intergeneracional: familia, profesionales de la atención sociosanitaria y la comunidad. De acuerdo con Gutiérrez (2011) persiguen beneficios individuales, sociales, culturales y económicos proporcionando oportunidades eficaces y eficientes. Los hallazgos de esta investigación señalan que la participación social de las personas mayores en la sociedad es fundamental para este colectivo y para las comunidades como vía de empoderamiento a nivel individual y comunitario.

Quinto hallazgo: Un proyecto sobre voluntariado virtual que promueve la participación social de las personas mayores de forma inclusiva e interactiva.

En este proyecto de intervención social los resultados señalan la mejora de la calidad de vida de las personas mayores mediante la implantación del voluntariado virtual. En la sociedad actual las TIC se han posicionado como palanca de cambio y están presentes en todos los ámbitos de la sociedad. Sin embargo, en el ámbito social tienen poco auge en comparación con otros sectores. Por otro lado, Colombo, Aroldi y Carlo (2015), ponen de manifiesto la existencia de la brecha digital en la población mayor en relación a la desigualdad de competencias y habilidades al acceso y uso de las TIC. Los hallazgos de esta investigación muestran la necesidad de impulsar la utilización de los recursos digitales que ofrecen las redes sociales a través del voluntariado digital para las personas mayores como forma de participación social e interacción en la sociedad.

Sexto hallazgo: Un proyecto de investigación cualitativa que analiza el impacto de los programas intergeneracionales desde la escuela y diferentes instituciones sociales.

Los resultados de esta investigación evidencian la satisfacción de las personas mayores por participar en programas intergeneracionales que tienen como objetivo empoderarse y seguir ampliando conocimientos. En relación a lo que se refiere a la esfera comunitaria, aparecen beneficios relacionados con la creación de redes sociales para mejorar el proceso de la integración social entre distintas generaciones (Granville y Hatton-Yeo, 2002; Sánchez, Díaz, López y Sáez, 2008; Gutiérrez y Hernández, 2013). También se incide en la importancia que tiene la consolidación de sociedades del futuro entendidas como espacios intergeneracionales de participación activa y educación social (Martínez de Miguel, Escarbajal y Moreno, 2017). Es fundamental que la información sobre las personas mayores llegue a la población joven para entender el proceso de envejecimiento sin prejuicios ni estereotipos negativos. Los hallazgos de esta investigación consideran que, a través de los programas intergeneracionales, se puede contribuir de forma eficaz y eficiente al desarrollo de la educación para la ciudadanía donde queden implicadas distintas generaciones. 


\section{Conclusiones}

Desde el trabajo social se considera que una manera eficaz para promover el envejecimiento activo y la participación social de las personas mayores debe ir acompañada del intercambio de experiencias entre distintas generaciones. En este sentido, el contacto directo entre diferentes generaciones produce numerosos beneficios para la persona.

Los programas intergeneracionales son un recurso idóneo para el trabajo social con la finalidad de conseguir mayores cotas de bienestar individual y social; en consecuencia, constituyen eficaces iniciativas de solidaridad que permiten empoderar a sus participantes Sin la cultura de convivencia intergeneracional se haría muy difícil alcanzar el tipo de solidaridad, cohesión social, participación social, inclusión y equidad como elementos importantes para hacer de nuestras sociedades espacios inclusivos para todas las edades.

Estos programas crean, en la comunidad, la integración de la población mayor con la población joven, la transmisión de conocimientos y experiencias y la consolidación del valor de las personas a través de la interacción que se produce entre ambas generaciones

En relación a los objetivos planteados en esta investigación se concluye exponiendo que, en los últimos cinco años, en España son escasos los estudios que han analizado, desde la disciplina del trabajo social, los beneficios que los programas intergeneracionales pueden tener para la población mayor, teniendo en cuenta la verdadera idiosincrasia que subyace en el concepto: "programa intergeneracional" y los verdaderos objetivos que se persigue en su aplicación.

Por lo tanto, y como propuesta de mejora sería importante recoger información tanto de los profesionales del trabajo social que se encargan de ponerlos en marcha como de otros agentes sociales que forman parte de la comunidad. Además, se necesita una mayor implicación institucional, social y científica para aprovechar al máximo las posibilidades que brindan la participación social de las personas mayores a través de los programas intergeneracionales.

En definitiva, un compromiso en esta área de investigación supone conseguir el empoderamiento de las personas mayores y avanzar hacia esa sociedad en la que tengan cabida todas las personas independientemente de su edad.

\section{Referencias}

Adams, R. 2008. Empowerment, participation and Social Work ( $3^{\mathrm{a}}$ ed.). New York: Palgrave Macmillan 
Blanco, A. 2016. Buenas prácticas del Proyecto Piloto Enred@te: red social digital para personas mayores y voluntariado de Cruz Roja Española. Cuadernos del Trabajo Social, (2), 201-212. https://doi.org/10.5209/CUTS.51656

Braveman, P., Egerter, S y Williams, D. 2011. The Social Determinants of Health: Coming of Age. Annual Review of Public Health, (32), 81-98. https://doi.org/10.1146/annurevpublhealth-031210-101218

Caballero, M y Artemio B. 2013. ¿Es operativo el concepto de generación? Aposta, (56), 1-45. Recuperado de http://www.apostadigital.com/revistav3/hemeroteca/mcg1.pdf

Carabaña, J. 2003. Los cambios demográficos y sus consecuencias sociales. ICE Revista de Economía, (811), 153-74. Recuperado de http://www.revistasice.com/index.php/ICE/ article/view/575

Colombo, F., Aroldi, P y Carlo, S. 2015. Nuevos mayores, viejas brechas: TIC, desigualdad y bienestar en la tercera edad en Italia. Comunicar, (45), 47-55. http://dx.doi.org/10.447/rasp.2017.2193

CSIC. 2019. Un perfil de las personas mayores en España, 2019 Indicadores estadísticos básicos. Recuperado de: http://envejecimiento.csic.es/documentos/documentos/ enred-indicadoresbasicos2019.pdf (14 de agosto de 2020)

Díaz, C., García, J y Nicasio, M. 2017. Online Gerontechnological Resources Analysis for Active Aging. Research on Ageing and Social Policy, (2), 155-198. http://dx.doi.org/10.447/rasp.2017.2193

Donati, P. P. 1999. Familias y generaciones. Desacatos, (2), 27-49. Recuperado de https://dialnet.unirioja.es/servlet/articulo?codigo $=5896579$

Escarbajal de Haro, A. y Martínez de Miguel, S. 2012. Jubilación, educación y calidad de vida. Pedagogía social. Revista Interuniversitaria, (20), 245-272. Recuperado de https://dialnet.unirioja.es/servlet/articulo?codigo=3975366

Eurostat. 2020. Estructura demográfica y envejecimiento de la población. Recuperado de: https://ec.europa.eu/eurostat/statistics-explained/index.php?title=Population_structure_and_ageing/es\#EI_porcentaje_de_personas_mayores_sigue_aumentando 104 de enero de 2021)

Faleiros, V. de P. 2003. Estrategias de empowerment en Trabajo Social. Buenos Aires: Lumen Humanitas.

Folgheraiter, F. 2012. Teoria e metodología del Servizio Sociale. La prospettiva de rete $4^{\mathrm{a}}$ ed). Milan: Franco Agnelli.

Freie Universität Berlín. 2021. Participación social. https://www.lai.fu-berlin.de/es/elearning/projekte/frauen_konzepte/projektseiten/konzeptebereich/rot_partizipacion/contexto/index.html (13 de mayo de 2021).

Granville, G. y Hatton-Yeo. 2002. Intergenerational engagement in the UK: A framework for creating inclusive communities. En M. Kaplan, N. Henkin, N. y A. Kusano (eds). Linking lifetimes: a global view of intergenerational. Exchange. Lanham, MD: University Press of América.

Gilabert, L y González, X. 2016. Los museos como factor de integración social del arte en la comunidad. La experiencia del voluntariado cultural de mayores. Cuadernos de Trabajo Social, (29), 83-93. https://doi.org/10.5209/rev_CUTS.2016.v29.n1.49247 
Gutiérrez, M y Hernández, D. 2013. Los beneficios de los programas intergeneracionales desde la perspectiva de los profesionales. Pedagogía Social. Revista Interuniversitaria, (21), 213-235. Recuperado de https://www.redalyc.org/articulo.oa?id=135029517010

Gobierno de España. 2019. Política Social. Recuperado de: https://www.lamoncloa.gob.es/ espana/eh18-19/sanidad/Paginas/psocial.aspx (04 de enero de 2021)

Goodman C. 1996. Literature Searching and. Evidence Interpretation for Assessing Health. Care Practices. Stockholm: SBU.

Gutiérrez, M. 2011. Programas intergeneracionales. Teoría, política y práctica. Saarbrücken: Editorial Académica Española.

Hank, K. 2012. Relaciones entre generaciones en Europa: una panorámica de las diferentes dimensiones de solidaridad familiar intergeneracional. Panorama Social, 15, 9-22. Recuperado de: https://dialnet.unirioja.es/servlet/articulo?codigo=3987808 (05 de enero de 2021).

Hanton-Yeo, A y Ohsako, T. 2001. Programas Intergeneracionales: Política pública e implicaciones de la investigación. Una perspectiva internacional. Hamburgo: UNESCO. Recuperado de https://www.infogerontologia.com/documents/gerontologia/envej_activo_ intergen/intergenspa.pdf

IMSERSO. 2011. Boletín sobre el envejecimiento. Perfiles y tendencias. Recuperado de http://www.imserso.es/InterPresent2/groups/imserso/documents/binario/boletinopm44. pdf (8 de diciembre de 2020).

IMSERSO. 2010. Programas Intergeneracionales. Guía introductoria. Recuperado de https://www.aepumayores.org/sites/default/files/programas_intergeneracionales_coleccion_manuales_guias_imserso_\%202010.pdf (7 de octubre de 2020).

IMSERSO. 2017. Estrategia Nacional de Personas Mayores para un Envejecimiento Activo y para su Buen Trato 2018-2021. Recuperado de https://www.algec.org/ wp-content/uploads/2017/12/Estrateg-Nacde-PM-2018-Imserso.pdf (14 de diciembre de 2020).

IMSERSO. 2018. Programas de envejecimiento activo. Recuperado de http://www.imserso.es/imserso_01/index.htm (14 de diciembre de 2020).

IMSERSO. 2018. Las personas mayores en España. Datos Estadísticos Estatales y por Comunidades Autónomas. (pp. 35-42) Recuperado de http://www.imserso.es/InterPresent1/groups/imserso/documents/binario/112017001_informe-2016-persona.pdf (16 de octubre de 2020).

INE. 2020. Cifras de población. Recuperado de https://www.ine.es/infografias/infografia_dia_poblacion.pdf 7 de octubre de 2020).

INE. 2020. La población en España crece hasta los 47,4 millones. Recuperado de https://www.Ine.es/sociedad/2020/04/21/poblacion-espana-crece-47-4-14641016.html (7 de octubre de 2020).

INE. 2020. Esperanza de vida en España. Recuperado de https://www.ine.es/ss/ Satellite? $\mathrm{L}=$ es_ES\&C $=$ INESeccion_C\&cid $=1259926380048 \& p=1254735110672 \&$ pagename=ProductosYServicios/PYSLayout\#: :text=En\%20Espa\%C3\%B1a\%20entre\%20 1999\%20y,b\%C3\%A1 sicos\%20que\%20publica\%20el\%20INE. (21 de septiembre de 2020).

Kuehne, V. S. 200). The state of our art: intergenerational program research and evaluation: part two. Journal of Intergenerational Relationships: Programs, Policy, and Research, (1), 79-94. https:// doi: 10.1300 / J194v01n02_07 
Laín, P. 1945. Las generaciones en la historia. Instituto de Estudios Políticos: Madrid. Recuperado de http://www.cervantesvirtual.com/obra/las-generaciones-en-la-historia/

Lambert, D., Dellmann-Jenkins, M. y Fruit, D. 1990. Planning for contact between the generations: An effective approach. The Gerontologist, (30), 553-556. http://dx.doi.org/10.1093/geront/30.4.553

Leccardi, C. y Carles F. 2011. El concepto de generación en las teorías sobre la juventud, Última Década, (34), 11-3. http://dx.doi.org/10.4067/S0718-22362011000100002

Lee, J. A. 2001. The empowerment approach to social work practice (2 ${ }^{a}$. ed.). New York: Columbia University Press.

MacCallum, J., Palmer, D., Wright, P., Cumming-Potvin, W., Northcote, J.,Booker, M. y Tero, C. 2006. Community building through intergenerational exchange programs. Australia: National Youth affairs Research Scheme.

McCreadie, C. y Tinker, A. 2005. The acceptability of assistive technology to older people, Ageing and Society, (1), 91-110. doi: 10.1017/S0144686X0400248X

Martin, M. 2008. La teoría de las generaciones de Ortega y Gasset: una lectura del siglo XXI, Tiempo y Espacio, (10), 98-110. Recuperado de http://www.ubiobio.cl/miweb/webfile/media/222/Tiempo/2008/07\%20Marco\%20Martin\%20articulo\%20pag\%2098-110.pdf

Martínez de Miguel, S. y Escarbajal de Haro, A. 2009. Alternativas socioeducativas para las personas mayores. Madrid: Dykinson.

Méndez, L. 2016. Arte y Trabajo Social: un programa de intervención para transformar la visión de la sociedad para la inclusión de personas con demencia temprana y Alzheimer. Cuadernos del Trabajo Social, (1), 51-61. https://doi.org/10.5209/rev_ CUTS.2016.v29.n1.49148

Moral, M. V. 2017. Programas intergeneracionales y participación social: la integración de los adultos mayores españoles y latinoamericanos en la comunidad. Universitas Psychologica, (1), 1-19. https://doi.org/10.11144/Javeriana.upsy16-1.pips

Moreno, P., Martínez de Miguel, S. y Escarbajal de Haro, A. 2018. El impacto educativo de los programas intergeneracionales: un estudio desde la escuela y las diferentes instituciones sociales implicadas. Revista Iberoamericana de Educación, (2), 31-54. https://doi.org/10.35362/rie7723158

Moher, D., Liberati, A., Tetzlaff, J., Altman, D.G y The PRISMA Group. 2009. Preferred reporting items for systematic reviews and meta-analyses: The PRISMA Statement. BMJ, 339, b2535. https://doi.org/10.1136/bmj.b2535

NACIONES UNIDAS. 2002. Informe de la Segunda Asamblea Mundial sobre el Envejecimiento. Nueva York: Naciones Unidas. Recuperado de https://social.un.org/ageing-working-group/documents/mipaa-sp.pdf

OMS. 2002. Envejecimiento activo: un marco político. Revista Española de Geriatría y Gerontología, (37), 74-105. Recuperado de https://www.elsevier.es/es-revista-revistaespanola-geriatria-gerontologia-124-articulo-envejecimiento-activo-un-marco-politico13035694

OMS. 2015. Informe Mundial sobre el Envejecimiento y la Salud. Recuperado de http://apps.who.int/iris/bitstream/handle/10665/186466/9789240694873_spa.pdf;jsession$\mathrm{id}=904142962 \mathrm{~B} 8$ AB33B84CE6F1362BC6F3A? sequence $=1$ (21 de septiembre de 2020) 
OMS. 2018. Envejecimiento y salud. Recuperado de https://www.who.int/es/ news-room/fact-sheets/detail/envejecimiento-y-salud (21 de septiembre de 2020)

Ostir, G.V; Ottenbacher, K.J. y Maarkides, K. S. 2004. Onset of frailty in older adults and the protective role of positive affect. Journal of Psychology and Aging, (3), 402-404. Recuperado de https://pubmed.ncbi.nlm.nih.gov/15382991/ (19/02/2021)

Mccrea, J. M; Weissman, M y Thorpe-Brown, G. 2004. Connecting the Generations: A Practical Guide for Developing Intergenerational Programs. Pittsburgh, PA: Generations Together.

Pinazo, S; Lorente, M.R; Limón L; Fernández, P y Bermejo F. 2010. Envejecimiento y aprendizaje a lo largo de la vida. Envejecimiento activo y actividades socioeducativas con personas mayores: guía de buenas prácticas, (2), 1-10. Recuperado de https://dialnet. unirioja.es/servlet/articulo?codigo $=4843139$

Pinazo, S; Kaplan, M. 2012. Las personas mayores proveedoras de conocimientos y cuidados. El papel de los programas intergeneracionales. Educación Social: Revista de Intervención Socioeducativa, (51), 45-66. Recuperado de https://dialnet.unirioja.es/ servlet/autor?codigo $=319335$

Sánchez, M; Díaz, P. 2005. Los programas intergeneracionales. Gerontología, innovación y propuestas. Madrid: Pearson Prentice Hall.

Serrat, R; Warburton, J; Petriwskyj, A; Villar, F. 2017. Political Participation and Late-Life Learning: A Cross-Cultural Study of Older People's Participation in Seniors' Interest Organisations in Australia and Spain. Voluntas: Baltimore.

Sixsmith, A. 2013. Technology and the challenge of aging. En A. Sixsmith y G. Gutman (eds.) Technologies for Active Aging (pp. 7-26). New York: Springer.

Ventura-Merkel, C. y Lidoff, L. 1983. Program innovation in aging: community planning for intergenerational programming. Washington D.C: National Council on Aging.

Walker, A. 2006. Active ageing in employment. Its meaning and potential. Asia-Pacific Review, 13 (1), 78-93. https://doi.org/10.1080/13439000600697621

Woolrych, R.; Gibson, N.; Sixsmith, J. y Sixsmith, A. 2015. "No Home, No Place»: Addressing the Complexity of Homelessness in Old Age Through Community Dialogue. Journal of Housing For the Elderly, (29), 93, 108, https;// doi: org/10.1080/02763893. 2015.1055024

Zafra E., Peiró R., Ramón, N., Álvarez-Dartel C y Borrell C. 2006. Análisis de las políticas sobre el envejecimiento en los planes autonómicos sociosanitarios y de atención a las personas mayores en España. Gac Sanit, (20), 295-302. http://dx.doi.org/10.4321/S1134928X2012000400002 


\section{comunitania}

REVISTA INTERNACIONAL DE TRABAJO SOCIAL Y CIENCIAS SOCIALES

INTERNATIONAL JOURNAL OF SOCIAL WORK AND SOCIAL SGIENCES

\section{ARTICULOSS/ARTICLES}

Violencia de género en la zona rural de la comarca de Alhama de Granada en 2015-2020 / Gender violence in the rural area of Alhama de Granada (2015-2020)

Cristiana Herreros Sánchez

La Seguridad Social costarricense frente el COVID-19: cuando la incertidumbre económica es peor que la incertidumbre epidemiológica / Costa Rican Social Security in the face of COVID-19: when economic uncertainty is worse than epidemiological uncertainty

Andrey Badilla Solano

Pobreza y voluntariado: viejos y nuevos retos / Poverty and volunteering: old and new challenges

Carolina Blavia Galindo

La solución de los países del norte de Europa a la pandemia del coronavirus. El aseguramiento y la colaboración público-privada / The way of the northern European countries to face the coronavirus pandemic. Assurance and public-private collaboration

Benito Cadenas Noreña

Programas intergeneracionales que fomentan la participación social de las personas mayores en España. Una mirada desde el Trabajo Social / Intergenerational programmes that promote the social participation of older people in Spain. A view from Social Work

Juana María Morcillo Martínez

Págs 103-118

\section{BESEIIAS/REVIIEWS}

Cristina Díaz, Verónica Giménez Béliveau, Marcelo Lucero y Washington Uranga (Coord.). 2020. Políticas Sociales. Estrategias para construir un nuevo horizonte de futuro / Social politics. Strategies to build a new horizon for the future. Buenos Aires: Ministerio de Desarrollo Social, CEIL-CONICET; RIPPSO; FAUATS (por Santiago Tafernaberry)

Bundschuh, S., Freitas, M. J., Palacín Bartrolí, C. and Žganec, N. (eds.). 2021. Ambivalences of Inclusion in Society and Social Work. Research-Based Reflections in Four European Countries / Ambivalencias de la inclusión en la sociedad y el trabajo social. Reflexiones basadas en la investigación en cuatro países europeos. London: Springer

(por Teresa Piaja Viñas) 